\title{
X-Ray Emission from Expanding Shells in NGC 3077
}

\author{
Jürgen Ott \\ CSIRO Australia Telescope National Facility, Cnr Vimiera \& Pembroke \\ Rds, Marsfield NSW 2122, Australia \\ Crystal L. Martin \\ University of California, Santa Barbara, Department of Physics, Santa \\ Barbara, CA 93106, USA \\ Fabian Walter \\ National Radio Astronomy Observatory, P.O. Box O, Socorro, NM \\ 87801, USA
}

\begin{abstract}
Deep Chandra observations of NGC 3077, a starburst dwarf galaxy in the M 81 triplet, resolve the X-ray emission from several supershells. The emission is brightest in the cavities defined by expanding shells detected previously in $\mathrm{H} \alpha$ emission. Thermal emission models fitted to the data imply temperatures ranging from 1.3 to $4.9 \times 10^{6} \mathrm{~K}$. The total $0.3-6.0 \mathrm{keV} \mathrm{X}$-ray luminosity is $2-5 \times 10^{39} \mathrm{erg} \mathrm{s}^{-1}$ (depending on the selected thermal plasma model). Most (85\%) of the X-ray luminosity in NGC 3077 comes from the hot interstellar gas; the remainder comes from six X-ray point sources. The radial density profile of the hot gas is not as steep as that expected in a freely expanding wind (e.g., as seen in the neighboring starburst galaxy M 82) implying that the hot gas is still confined by the $\mathrm{H} \alpha$ shells.
\end{abstract}

\section{Introduction}

Starburst phenomena in dwarf galaxies may play a key role in the metal enrichment of the intergalactic medium. Hierarchical models of structure formation predict that larger galaxies assemble from smaller systems such as dwarf galaxies. For this reason, dwarf galaxies which are the most numerous type of galaxy in the local universe must have been even more abundant at larger lookback times. Due to their low gravitational potential, a galactic wind developing from the mechanical energy input of stellar winds and type II supernovae can transport metal-enriched material more easily to the intergalactic medium as compared to more massive galaxies (see, e.g., MacLow \& Ferrara 1999; Ferrara \& Tolstoy 2000; Silich \& Tenorio-Tagle 2001; Martin 1998; Martin, Kobulnicky, \& Heckman 2002). 


\section{Chandra Observations of NGC 3077}

The mechanical energy released in a starburst is converted into hot gas with temperatures of order $10^{6-7} \mathrm{~K}$ which can be observed by thermal X-ray emission. We obtained spectral imaging data of the nearby dwarf starburst galaxy NGC 3077 observed with the Chandra X-ray observatory (ACIS-S3). The data analysis and our results are described in Ott, Martin, \& Walter (2003). Previously, Martin (1998) detected a number of expanding shells in $\mathrm{H} \alpha$ emission. The Chandra data reveal the presence of hot gas with temperatures of 1.3 to $4.9 \times 10^{6} \mathrm{~K}$ which is nicely associated with the interior of these shells. This allows us to compare the hot gas properties of different shells within a single galaxy (Ott, Martin, \& Walter 2003). The hot gas within the individual shells exhibits pressures of $P / k \approx 10^{5-6} \mathrm{~K} \mathrm{~cm}^{-3}$ which is about two orders of magnitude higher than the typical pressure in the interstellar medium. The hot gas is therefore likely to be responsible for the expansion of the cavities.

Theoretical models (Chevalier \& Clegg 1985) for a spherically-symmetric freely flowing wind predict a power law in the radial volume density distribution of the hot gas with an index of -2 . This is not consistent with our data which, in contrast, suggest an exponential decline. This is an indication that NGC 3077 has not developed large-scale outflows, yet. However, the gas is hot enough to overcome the gravitational potential of NGC 3077 and may flow out in the future (as compared to the calculations of Silich \& Tenorio-Tagle 2001). Toward the south of NGC 3077 , however, a massive tidal tail is present, visible in the line of neutral hydrogen (see Yun, Ho, \& Lo 1994; Walter et al. 2002). This feature appears to act as a "barrier" for future outflows. Indeed, the X-ray emission of NGC 3077 is elongated toward the opposite of the tidal tail. Outflow is therefore quite unlikely along the southern direction of NGC 3077 but is still possible toward the north.

In addition to the diffuse X-ray emission within the shells of NGC 3077, an additional $\sim 30 \%$ cannot be attributed to the individual shells; $15 \%$ of the total X-ray luminosity of NGC $3077\left(2-5 \times 10^{39} \mathrm{erg} \mathrm{s}^{-1}\right)$ is emitted by six point sources which comprise three supernova remnants, two $\mathrm{X}$-ray binaries and a supersoft source.

\section{References}

Chevalier, R. A. \& Clegg, A. W. 1985, Nature, 317, 44

Ferrara, A. \& Tolstoy, E. 2000, MNRAS, 313, 291

Mac Low, M. \& Ferrara, A. 1999, ApJ, 513, 142

Martin, C. L. 1998, ApJ, 506, 222

Martin, C., Kobulnicky, H., \& Heckman, T. 2002, ApJ, 574, 663

Ott, J., Martin, C. L., Walter, F. 2003, ApJ, 594, 776

Silich, S. \& Tenorio-Tagle, G. 2001, ApJ, 552, 91

Walter, F., Weiss, A., Martin, C., \& Scoville, N. 2002, AJ, 123, 225

Yun, M. S., Ho, P. T. P., \& Lo, K. Y. 1994, Nature, 372, 530 